\title{
Geometric Cauchy-Schwarz inequality
}

\author{
Gen WANG* \\ School of Mathematical Sciences, Xiamen University, \\ Xiamen, 361005, P.R.China.
}

\begin{abstract}
In this paper, using the generalized geometric commutator and geomutator to develop the geometric anticommutator and anti-geomutator, then we strictly prove geometric Cauchy-Schwarz inequality in quantum method as a generalization of the Cauchy-Schwarz inequality based on the Hermitian operator. It certainly demonstrates a fact that environment variable is unavoidable for the revision of the Cauchy-Schwarz inequality for the quantum mechanics.
\end{abstract}

\section{Contents}

$\begin{array}{llr}1 & \text { Introduction } & 1\end{array}$

1.1 Cauchy-Schwarz inequality . . . . . . . . . . . . . . . . 2

1.2 Formulations of the uncertainty relation . . . . . . . . . . . . 2

\begin{tabular}{|lll}
2 & Generalized geometric commutator and geometric anticommutator & 3
\end{tabular}

2.1 The GGC and the GAC . . . . . . . . . . . . . 4

$2.2 \quad$ QCPB and quantum geobracket (QGB) . . . . . . . . . . . . . 4

\begin{tabular}{|lll}
3 & Geometric Cauchy-Schwarz inequality & $\mathbf{5}$
\end{tabular}

3.1 The generalized variance and geometric operator . . . . . . . . . . . 5

3.2 Geometric Cauchy-Schwarz inequality . . . . . . . . . . . . . 6

$3.3 \quad$ Geometric entanglement term . . . . . . . . . . . . . . . . . 9

\section{Introduction}

The Cauchy-Schwarz inequality [1] as a useful inequality used in many different fields, such as linear algebra, analysis, probability theory, vector algebra and other areas. It is seen as the one of the most important inequalities in all of mathematics. The inequality for sums was published by Cauchy in 1821s, while the corresponding

* Sterces ofo neila ygoloncet, phd. email: Wg1991dream@163.com 


\section{G. WANG}

inequality for integrals was first proved by Bunyakovsky in 1859s. Various generalizations [2] of the Cauchy-Schwarz inequality exist. Hölder's inequality generalizes it to $L^{p}$ norms. More generally, it can be interpreted as a special case of the definition of the norm of a linear operator on a Banach space. Further generalizations can be seen from the field of operator theory [3].

The Cauchy-Schwarz inequality has widely used in quantum mechanics to prove the generalized uncertainty relation, ect. As quantum mechanics shown, the most fundamental features of quantum mechanics is the existence of nontrivial error-disturbance relations for quantum measurements. With the non-universal validity of the QPB that has been replaced by the complete QCPB, a series of changes about the QPB will follow for sure. This paper uses the quantum method on the Hermitian operator to get deduced the geometric Cauchy-Schwarz inequality.

\subsection{Cauchy-Schwarz inequality}

The Cauchy-Schwarz inequality states that for all vectors $\mathbf{u}$ and $\mathbf{v}$ of an inner product space it is true that

$$
|\langle\mathbf{u}, \mathbf{v}\rangle|^{2} \leq\langle\mathbf{u}, \mathbf{u}\rangle \cdot\langle\mathbf{v}, \mathbf{v}\rangle,
$$

where $\langle\cdot, \cdot\rangle$ is the inner product. Examples of inner products include the real and complex dot product. Equivalently, by taking the square root of both sides, and referring to the norms of the vectors, the inequality is written as $|\langle\mathbf{u}, \mathbf{v}\rangle| \leq\|\mathbf{u}\|\|\mathbf{v}\|$. Moreover, the two sides are equal if and only if $\mathbf{u}$ and $\mathbf{v}$ are linearly dependent.

\subsection{Formulations of the uncertainty relation}

The relation between the error and disturbance in quantum measurements was first studied by Heisenberg. Introducing his famous uncertainty principle, he derived a trade-off relation between the error $\epsilon(X)$ of the position measurement and the thereby caused momentum disturbance $\eta(P)$ given by [4]

$$
\epsilon(X) \eta(P) \geq \hbar / 2
$$

However, by the development of the theory of quantum measurement, it was revealed that Heisenberg's relation does not hold in general.

There are some general form of formulations of the uncertainty relation. The Robertson uncertainty relation is generally formulated as the relation [5,6]

$$
\sigma(A, \psi) \sigma(B, \psi) \geq \frac{\left|\left\langle\psi\left|[A, B]_{Q P B}\right| \psi\right\rangle\right|}{2}
$$

for any observables $A, B$ and any state $\psi$, where the standard deviation $\sigma(X, \psi)$ of an observable $X$ in state $\psi$ is defined by

$$
\sigma^{2}(X, \psi)=\left\langle\psi\left|X^{2}\right| \psi\right\rangle-\langle\psi|X| \psi\rangle^{2}
$$

This relation was proven mathematically from fundamental postulates of quantum mechanics. Nevertheless, this relation describes the limitation on preparing microscopic objects but has no direct relevance to the limitation of accuracy of measuring devices. 


\section{G. WANG}

The Robertson uncertainty relation immediately follows from a slightly stronger inequality — the Schrödinger uncertainty relation that is given by 7,8

$$
\sigma(A, \psi) \sigma(B, \psi) \geq \sqrt{\left(\frac{1}{2}\left\langle\{A, B\}_{i r}\right\rangle-\langle A\rangle\langle B\rangle\right)^{2}+\left(\frac{1}{2 \sqrt{-1}}\left\langle[A, B]_{Q P B}\right\rangle\right)^{2}}
$$

As a result, the QCPB [9] should be a substitute for all the QPB as a core in the above uncertainty relation,

$$
\begin{aligned}
|\langle[A, B]\rangle| / 2 & =\left|\left\langle[A, B]_{Q P B}+G(s, A, B)\right\rangle\right| / 2 \\
& =\left|\left\langle[A, B]_{Q P B}\right\rangle+\langle G(s, A, B)\rangle\right| / 2
\end{aligned}
$$

As it shown, the quantum geometric bracket $G(s, A, B)$ occupies a large proportion in the formula which contains the environment variable.

\section{Generalized geometric commutator and geo- metric anticommutator}

Let $a$ and $b$ be any two operators, their commutator is formally defined by

$$
[a, b]_{c r}=a b-b a .
$$

Let $a$ and $b$ be any elements of any algebra, or any operators, their generalized geometric commutator is formally defined by the following.

Definition 1 (GGC). [9] A generalized geometric commutator (GGC) of arity two $a, b$ is formally given by

$$
[a, b]=[a, b]_{c r}+G(s ; a, b)
$$

The geomutator is

$$
G(s ; a, b)=a[s, b]_{c r}-b[s, a]_{c r}
$$

satisfying $G(s ; a, b)=-G(s ; b, a)$, where $s$ is a geometric structure function given by domain.

Obviously, in some sense, the anticommutator also needs to be generalized as the commutator does, Analogously, denote by $\{a, b\}_{i r}=a b+b a$ the anticommutator,

Definition 2. The geometric anticommutator $(G A C)$ of any two elements $a$ and $b$ is defined by

$$
\{a, b\}=\{a, b\}_{i r}+Z(s, a, b)
$$

where $Z(s, a, b)=Z(s, b, a)$ is called anti-geomutator, $s$ is geometric function.

As the definition above stated,

Definition 3. The anti-geomutator can be taken as

$$
Z(s, a, b)=(a: s: b)+\{a, b\}_{i r} s
$$

where $(a: s: b)=a s b+b s a$, and $s$ is the geometric function created by the environment. 


\section{G. WANG}

Note that in order to preserve the related property of the anticommutator, we always let anti-geomutator $Z(s, a, b)$ satisfiy the symmetry, namely,

$$
Z(s, a, b)=Z(s, b, a)
$$

As a result of the symmetry of anti-geomutator, geometric anticommutator then follows the symmetry $\{a, b\}=\{b, a\}$. Actually, the anti-geomutator can be expressed in the form

$$
\begin{aligned}
Z(s, a, b) & =a\{s, b\}_{i r}+b\{s, a\}_{i r} \\
& =a s b+b s a+a b s+b a s \\
& =(a: s: b)+\{a, b\}_{i r} s
\end{aligned}
$$

As seen, this form is very similar to the quantum geometric bracket, this is why we need to generalize the anticommutator.

\subsection{The GGC and the GAC}

In conclusions, the generalized geometric commutator (GGC) and the geometric anticommutator (GAC) can be listed as below

$$
\begin{aligned}
& \text { The GGC and the GAC: } \\
& \\
& {[a, b]=[a, b]_{c r}+G(s, a, b),} \\
& \{a, b\}=\{a, b\}_{i r}+Z(s, a, b),
\end{aligned}
$$

Meanwhile, the geomutator and anti-geomutator then follow

$$
\begin{aligned}
G(s, a, b) & =a[s, b]_{c r}-b[s, a]_{c r} \\
Z(s, a, b) & =a\{s, b\}_{i r}+b\{s, a\}_{i r}
\end{aligned}
$$

\subsection{QCPB and quantum geobracket (QGB)}

As [9] stated, quantum covariant Poisson bracket (QCPB) is defined by generalized geometric commutator (GGC) while quantum geometric bracket (QGB) is given based on the geomutator. More precisely,

Definition 4 (QCPB). [9] The QCPB is generally defined as

$$
[f, g]=[f, g]_{Q P B}+G(s, f, g)
$$

in terms of quantum operator $f, g$, where $G(s, f, g)=-G(s, g, f)$ is called quantum geometric bracket.

It is zero if and only if $f$ and $g$ covariant commute, i,e. $[f, g]=0$. It is remarkable to see that the QCPB representation admits a dynamical geometric bracket formula on the manifold. Note that structural function $s$ represents the background property of spacetime. 
Definition 5 (Quantum geometric bracket (QGB)). [9] The quantum geometric bracket is

$$
G(s, f, g)=f[s, g]_{Q P B}-g[s, f]_{Q P B}
$$

where $s$ represents the globally condition of space.

Let's assert the role of the structure function $s$ that is a geometric structure function given by domain based on the generalized geometric commutator, it means that structure function $s$ is only determined by the environment, or spacetime, or manifolds, the domain, ect, from this viewpoint, the environment joins the physical process, the influence of the environment now based on new theory can't be ignored, it's naturally necessary to be considered in a physical process. In other words, the environment has joined the physical process, accordingly. By using the antisymmetric, the quantum geometric bracket (QGB) is rewritten as

$$
G(s, f, g)=f[s, g]_{Q P B}+g[f, s]_{Q P B}
$$

This expression can vividly and concretely state that the environment interacts with the operators $f, g$ respectively.

\section{Geometric Cauchy-Schwarz inequality}

\subsection{The generalized variance and geometric operator}

Definition 6 (Geoperator). Let $X$ be a Hermitian operator, then geoperator can be defined as

$$
X^{(s)}=X+u
$$

where $u=X s=I(X, s)$ is coupling interaction between the observable $X$ and the environment $s$.

Obviously, the geometric operator is a extension of the Hermitian operator. Note that the interaction term $u=X s \neq 0$ actually is a precise function with respect to the spacetime, as we state, the structure function $s$ generated by the space or manifolds as an environment variable only associate with the space or the manifolds, and it's independent to the wave function.

According to (2), based upon the definition of variance, the generalized variance $\Sigma^{2}(X, \psi)$ of an observable $X$ in state $\psi$ is given by

$$
\begin{aligned}
\Sigma^{2}(X, \psi) & =\left\langle\psi\left|X^{(s)^{2}}\right| \psi\right\rangle-\left\langle\psi\left|X^{(s)}\right| \psi\right\rangle^{2} \\
& =\left\langle\psi\left|(X+u)^{2}\right| \psi\right\rangle-\langle\psi|(X+u)| \psi\rangle^{2} \\
& =\left\langle\psi\left|(X+u)^{2}\right| \psi\right\rangle-(\langle\psi|X| \psi\rangle+\langle\psi|u| \psi\rangle)^{2} \\
& =\left\langle\psi\left|X^{2}+u^{2}+X u+u X\right| \psi\right\rangle-(\langle\psi|X| \psi\rangle+\langle\psi|u| \psi\rangle)^{2} \\
& =\left\langle\psi\left|X^{2}\right| \psi\right\rangle-\langle\psi|X| \psi\rangle^{2}+\rho(X, u) \\
& =\sigma^{2}(X, \psi)+\rho(X, s)
\end{aligned}
$$

where entanglement term $\rho(X, s)$ of an observable $X$ in state $\psi$ is given by

$$
\rho(X, s)=\left\langle\psi\left|u^{2}+X u+u X\right| \psi\right\rangle-\langle\psi|u| \psi\rangle^{2}-2\langle\psi|X| \psi\rangle\langle\psi|u| \psi\rangle
$$




$$
\begin{aligned}
& =\left\langle\psi\left|u^{2}\right| \psi\right\rangle-\langle\psi|u| \psi\rangle^{2}+\left\langle\psi\left|\{X, u\}_{i r}\right| \psi\right\rangle-2\langle\psi|X| \psi\rangle\langle\psi|u| \psi\rangle \\
& =\sigma^{2}(u, \psi)-2\langle\psi|X| \psi\rangle\langle\psi|u| \psi\rangle+\left\langle\psi\left|\{X, u\}_{i r}\right| \psi\right\rangle
\end{aligned}
$$

\subsection{Geometric Cauchy-Schwarz inequality}

In this paper, the mean values for any observables can be expressed as

$$
\langle X\rangle=\bar{X}=\langle\psi|X| \psi\rangle
$$

Let's always suppose that the Hermitian operator $X$ is not an angle variable and $Y$ is not the derivative in terms of this angle variable in this paper. And the $Y \psi$ is in the domain of the unbounded operator $X$ in the most situations.

Theorem 1. Geometric Cauchy-Schwarz inequality is

$$
\Sigma^{2}(X, \psi) \Sigma^{2}(Y, \psi) \geq{\overline{\Delta X^{(s)} \Delta Y^{(s)}}}^{2}
$$

for any Hermitian operator $X$ and $Y$.

Proof. By using the geoperator in the definition 6, we define the generalized variance $\Sigma^{2}(X, \psi)(3)$ of an observable $X$ in state $\psi$. In another way,

$$
\begin{aligned}
\Delta X^{(s)} & =X^{(s)}-\overline{X^{(s)}}=X+u-\overline{X+u} \\
& =X-\bar{X}+u-\bar{u}=\Delta X+\Delta u
\end{aligned}
$$

and for $Y^{(s)}$, is written out explicitly as

$$
\begin{aligned}
\Delta Y^{(s)} & =Y^{(s)}-\overline{Y^{(s)}}=Y+v-\overline{Y+v} \\
& =Y-\bar{Y}+v-\bar{v}=\Delta Y+\Delta v
\end{aligned}
$$

where $u=X s, v=Y s$. Then it gets

$$
\begin{aligned}
\Delta X^{(s)} \Delta Y^{(s)} & =\Delta X \Delta Y+\Delta u \Delta Y+\Delta X \Delta v+\Delta u \Delta v \\
& =X Y-\bar{X} Y-X \bar{Y}+\overline{X Y}+u Y-\bar{u} Y \\
& -u \bar{Y}+\bar{u} \bar{Y}+X v-\bar{X} v-X \bar{v} \\
& +\bar{X} \bar{v}+u v-\bar{u} v-u \bar{v}+\overline{u v}
\end{aligned}
$$

Similarly, we have

$$
\begin{aligned}
\Delta Y^{(s)} \Delta X^{(s)} & =\Delta Y \Delta X+\Delta v \Delta X+\Delta Y \Delta u+\Delta v \Delta u \\
& =Y X-\bar{Y} X-Y \bar{X}+\overline{X Y}+v X-\bar{v} X \\
& -v \bar{X}+\bar{v} \bar{X}+Y u-\bar{Y} u-Y \bar{u} \\
& +\bar{Y} \bar{u}+v u-\bar{v} u-v \bar{u}+\overline{v u}
\end{aligned}
$$

where

$$
\begin{aligned}
& \Delta X \Delta Y=X Y-\bar{X} Y-X \bar{Y}+\overline{X Y} \\
& \Delta u \Delta Y=u Y-\bar{u} Y-u \bar{Y}+\bar{u} \bar{Y}
\end{aligned}
$$




$$
\begin{aligned}
& \Delta X \Delta v=X v-\bar{X} v-X \bar{v}+\bar{X} \bar{v} \\
& \Delta u \Delta v=u v-\bar{u} v-u \bar{v}+\overline{u v}
\end{aligned}
$$

Therefore, it yields

$$
\begin{aligned}
& {\left[\Delta X^{(s)}, \Delta Y^{(s)}\right]_{Q P B}=[X, Y]+2[X, Y]_{Q P B} s} \\
& +\bar{Y} X+Y \bar{X}+\bar{v} X+v \bar{X}+\bar{Y} u+Y \bar{u} \\
& -\bar{X} Y-X \bar{Y}-\bar{u} Y-u \bar{Y}-\bar{X} v-X \bar{v}
\end{aligned}
$$

where $[X, Y]=[X, Y]_{Q P B}+G(s, X, Y)$ is the QCPB, and $G(s, X, Y)=\langle X: s: Y\rangle-$ $[X, Y]_{Q P B} s$ is the quantum geometric bracket.

Meanwhile,

$$
\begin{aligned}
& \left\{\Delta X^{(s)}, \Delta Y^{(s)}\right\}_{i r}=\{X, Y\}+2 u v+2 \overline{X Y}+2 \bar{u} \bar{Y}+2 \bar{X} \bar{v}+2 \overline{u v} \\
& -\bar{X} Y-X \bar{Y}-\bar{u} Y-u \bar{Y}-\bar{X} v-X \bar{v}-\bar{u} v-u \bar{v} \\
& -\bar{Y} X-Y \bar{X}-\bar{v} X-v \bar{X}-\bar{Y} u-Y \bar{u}-\bar{v} u-v \bar{u}
\end{aligned}
$$

where $\{X, Y\}=\{X, Y\}_{i r}+Z(s, X, Y)$ is the geometric anticommutator, $Z(s, X, Y)=$ $(X: s: Y)+\{X, Y\}_{i r} s$ is the anti-geomutator.

It follows the expectation value in terms of the antisymmetric part

$$
\begin{aligned}
& \left\langle\psi\left|\left[\Delta X^{(s)}, \Delta Y^{(s)}\right]_{Q P B}\right| \psi\right\rangle \\
& =\left\langle\psi\left|[X, Y]+2[X, Y]_{Q P B} s\right| \psi\right\rangle \\
& =\overline{[X, Y]}+2 \overline{[X, Y]_{Q P B} s}
\end{aligned}
$$

while the symmetric part is given by

$$
\left\langle\psi\left|\left\{\Delta X^{(s)}, \Delta Y^{(s)}\right\}_{i r}\right| \psi\right\rangle=\overline{\{X, Y\}}+2(\overline{u v}-(\bar{Y}+\bar{v})(\bar{X}+\bar{u}))
$$

Above all, we have

$$
\begin{aligned}
& \Delta X^{(s)} \Delta Y^{(s)}=\frac{1}{2}\left[\Delta X^{(s)}, \Delta Y^{(s)}\right]_{Q P B}+\frac{1}{2}\left\{\Delta X^{(s)}, \Delta Y^{(s)}\right\}_{i r} \\
& =\frac{1}{2}\left\{\Delta X^{(s)}, \Delta Y^{(s)}\right\}_{i r}+\sqrt{-1} \frac{\left[\Delta X^{(s)}, \Delta Y^{(s)}\right]_{Q P B}}{2 \sqrt{-1}}
\end{aligned}
$$

As a result,

$$
\begin{gathered}
\left\langle\psi\left|\Delta X^{(s)} \Delta Y^{(s)}\right| \psi\right\rangle=\left(\frac{\overline{\{X, Y\}}}{2}+\overline{u v}-(\bar{Y}+\bar{v})(\bar{X}+\bar{u})\right) \\
+\sqrt{-1}\left(\frac{\overline{[X, Y]}+2 \overline{[X, Y]_{Q P B} s}}{2 \sqrt{-1}}\right)
\end{gathered}
$$

or in the form

$$
\overline{\Delta X^{(s)} \Delta Y^{(s)}}=\left(\frac{\overline{\{X, Y\}}}{2}+\overline{u v}-(\bar{Y}+\bar{v})(\bar{X}+\bar{u})\right)+\sqrt{-1}\left(\frac{\overline{[X, Y]}+2 \overline{[X, Y]_{Q P B} s}}{2 \sqrt{-1}}\right)
$$


and

$$
\overline{\Delta X^{(s)} \Delta Y^{(s)}}=\left(\frac{\overline{\{X, Y\}}}{2}+\theta(s, X, Y)\right)+\sqrt{-1}\left(\overline{\frac{[X, Y]}{2 \sqrt{-1}}}+\vartheta(s, X, Y)\right)
$$

where

$$
\begin{aligned}
& \theta(s, X, Y)=\overline{u v}-(\bar{Y}+\bar{v})(\bar{X}+\bar{u}) \\
& \vartheta(s, X, Y)=-\sqrt{-1}[X, Y]_{Q P B} s
\end{aligned}
$$

Then, it leads to

$$
\left|\overline{\Delta X^{(s)} \Delta Y^{(s)}}\right|^{2}=\left(\frac{\overline{\{X, Y\}}}{2}+\theta(s, X, Y)\right)^{2}+\left(\frac{\overline{[X, Y]}}{2 \sqrt{-1}}+\vartheta(s, X, Y)\right)^{2}
$$

For the sake of argument that we manage to separate the classical theory

$$
\begin{aligned}
& \overline{\left[\Delta X^{(s)}, \Delta Y^{(s)}\right]_{Q P B}}=\overline{[X, Y]_{Q P B}}+\overline{G(s, X, Y)}+2 \sqrt{-1} \vartheta(s, X, Y) \\
& \overline{\left\{\Delta X^{(s)}, \Delta Y^{(s)}\right\}_{i r}}=\overline{\{X, Y\}_{i r}}+\overline{Z(s, X, Y)}+2 \theta(s, X, Y)
\end{aligned}
$$

Thusly, we get

$$
\begin{aligned}
\overline{\Delta X^{(s)} \Delta Y^{(s)}}= & \frac{1}{2} \overline{\left\{\Delta X^{(s)}, \Delta Y^{(s)}\right\}_{i r}}+\frac{1}{2} \overline{\left[\Delta X^{(s)}, \Delta Y^{(s)}\right]_{Q P B}} \\
= & \left(\frac{1}{2} \overline{\{X, Y\}_{i r}}+\frac{1}{2} \overline{Z(s, X, Y)}+\theta(s, X, Y)\right) \\
+ & \sqrt{-1} \overline{[X, Y]_{Q P B}}+\overline{G(s, X, Y)}+2 \sqrt{-1} \vartheta(s, X, Y) \\
= & \left(\frac{1}{2} \overline{\{X, Y\}_{i r}}+\frac{1}{2} \overline{Z(s, X, Y)}+\theta(s, X, Y)\right) \\
& +\sqrt{-1}\left(\frac{[X, Y]_{Q P B}}{2 \sqrt{-1}}+\frac{\overline{G(s, X, Y)}}{2 \sqrt{-1}}+\overline{\vartheta(s, X, Y)}\right)
\end{aligned}
$$

Then the square form is given by

$$
\begin{gathered}
{\overline{\Delta X^{(s)} \Delta Y^{(s)}}}^{2}=\left(\frac{1}{2} \overline{\{X, Y\}_{i r}}+\frac{1}{2} \overline{Z(s, X, Y)}+\theta(s, X, Y)\right)^{2} \\
+\left(\frac{\overline{[X, Y]_{Q P B}}}{2 \sqrt{-1}}+\frac{\overline{G(s, X, Y)}}{2 \sqrt{-1}}+\overline{\vartheta(s, X, Y)}\right)^{2}
\end{gathered}
$$

Therefore,

$$
\Sigma^{2}(X, \psi) \Sigma^{2}(Y, \psi) \geq{\overline{\Delta X^{(s)} \Delta Y^{(s)}}}^{2}
$$




\subsection{Geometric entanglement term}

Based on the generalized variance $\Sigma^{2}(X, \psi)$ of an observable $X$ in state $\psi$, we give the concept of the geometric entanglement term as follows.

According to (11), the product between the generalized variance $\Sigma^{2}(A, \psi)$ and $\Sigma^{2}(B, \psi)$ for any observables $A, B$ and any state $\psi$ is $\Sigma^{2}(A, \psi) \Sigma^{2}(B, \psi)$ that generally formulated as

$$
\begin{aligned}
& \Sigma^{2}(A, \psi) \Sigma^{2}(B, \psi) \\
& =\left(\sigma^{2}(A, \psi)+\rho(A, s)\right)\left(\sigma^{2}(B, \psi)+\rho(B, s)\right) \\
& =\sigma^{2}(A, \psi) \sigma^{2}(B, \psi)+\Theta(s, A, B)
\end{aligned}
$$

where the geometric entanglement term is given by

$$
\begin{aligned}
& \Theta(s, A, B)=\rho(A, s) \sigma^{2}(B, \psi) \\
& \quad+\sigma^{2}(A, \psi) \rho(B, s)+\rho(A, s) \rho(B, s)
\end{aligned}
$$

and $\sigma^{2}(A, \psi) \sigma^{2}(B, \psi)$ corresponds to the classical Cauchy-Schwarz inequality for quantum mechanics. It evidently says that the geometric entanglement term is the cause of the inevitability of environment. Hence,

$$
\Sigma^{2}(A, \psi) \Sigma^{2}(B, \psi)=\sigma^{2}(A, \psi) \sigma^{2}(B, \psi)+\Theta(s, A, B, \psi)
$$

As a result of the complete expression given by (6) with the geometric entanglement term expressed as

$$
\Theta(s, A, B)=\Sigma^{2}(A, \psi) \Sigma^{2}(B, \psi)-\sigma^{2}(A, \psi) \sigma^{2}(B, \psi)
$$

It implies that classic result $\sigma^{2}(A, \psi) \sigma^{2}(B, \psi)$ has to be revised by using new form $\Sigma^{2}(A, \psi) \Sigma^{2}(B, \psi)$.

\section{References}

[1] Steele, J. Michael. The Cauchy-Schwarz Master Class: an Introduction to the Art of Mathematical Inequalities. The Mathematical Association of America. 2004, p. 1.

[2] Wu, Hui-Hua; Wu, Shanhe. Various proofs of the Cauchy-Schwarz inequality. Octogon Mathematical Magazine. 2009, 17 (1): 221-229.

[3] Kadison, Richard V. A Generalized Schwarz Inequality and Algebraic Invariants for Operator Algebras. Annals of Mathematics. 1952, 56 (3): 494-503.

[4] Heisenberg W 1927 Z. Phys. 43 172. [English translation by J. A. Wheeler and W. H. Zurek, in Quantum Theory and Measurement, edited by J. A. Wheeler and W. H. Zurek (Princeton UP, Princeton, NJ, 1983), pp. 62-84].

Werner Heisenberg, The Physical Principles of the Quantum Theory, p. 20

[5] Kennard E H 1927 Z. Phys. 44326.

[6] Weyl H 1928 Gruppentheorie und Quantenmechanik (Leipzig: Hirzel Verlag) 
[7] Robertson H P 1929 Phys. Rev. 34163.

Robertson H P 1930 Phys. Rev. 35 667A.

Robertson H P 1934 Phys. Rev. 46794.

[8] Schrödinger E 1930 Sitz. Preus. Acad. Wiss. (Phys.-Math. Klasse) 296

[9] G Wang. Generalized geometric commutator theory and quantum geometric bracket and its uses [J]. arXiv:2001.08566

[10] G Wang. A revision for Heisenberg uncertainty relation based on environment variable in the QCPB theory [J]. arXiv:2003.07203. 